\title{
Review
}

\section{Nanoindentation of Historic and Artists' Paints}

\author{
Michał Łukomski *D, Alexandra Bridarolli and Naoki Fujisawa
}

check for updates

Citation: Łukomski, M.; Bridarolli, A.; Fujisawa, N. Nanoindentation of Historic and Artists' Paints. Appl. Sci. 2022, 12, 1018. https://doi.org/ 10.3390/app12031018

Academic Editor: Emanuela Bosco

Received: 24 December 2021

Accepted: 14 January 2022

Published: 19 January 2022

Publisher's Note: MDPI stays neutral with regard to jurisdictional claims in published maps and institutional affiliations.

Copyright: (c) 2022 by the authors. Licensee MDPI, Basel, Switzerland. This article is an open access article distributed under the terms and conditions of the Creative Commons Attribution (CC BY) license (https:/ / creativecommons.org/licenses/by/ $4.0 /)$.
Science Department, The Getty Conservation Institute, 1200 Getty Center Drive, Suite 700, Los Angeles, CA 90049, USA; al.bridarolli@gmail.com (A.B.); nfujisawa@getty.edu (N.F.)

* Correspondence: mlukomski@getty.edu

\begin{abstract}
The micromechanical analysis of composite materials has become indispensable in the field of material science and for industrial applications, where small sample sizes predominate. In recent years, nanoindentation has been more readily utilized within the cultural heritage field, producing invaluable insight in the properties of artists' materials. To take full advantage of the technique, however, issues related to the scale of the analysis need to be addressed. These include understanding the influence of sample preparation and material heterogeneity on the obtained results as well as establishing correlations between micro/nano-scale and macro-scale mechanical parameters. Moreover, the nanoindentation tests of time-dependent paints and adhesives can be difficult to interpret and thus require the development of unique measuring protocols. This review discusses nanoindentation studies of artists' paints carried out over the last two decades. Analysis of presented experimental and theoretical works focuses on comprehending limitations of the technique and developing strategies to overcome them. We demonstrate how consistent and reliable measurements can be performed when experimental protocols consider the effects of geometry, roughness, and time-dependent properties of paint, as well as the compliance of the measuring system. Development of measurement protocols accounting for specific properties of historic and artists' paints opens up the prospect of more routine application of nanoindentation in the field of cultural heritage.
\end{abstract}

Keywords: nanoindentation; historic paints; artists' paints; mechanical properties; micromechanics

\section{Introduction}

Mechanical characterization of materials constituting works of art is crucial for understanding the environmentally-induced risk of damage and developing passive and active conservation strategies [1]. However, in the heritage conservation field, the standard approach is to limit sampling to the absolute minimum. This disqualifies conventional tensile and compression tests that require the destruction of macroscopic sample sizes. To avoid the use of large amounts of historic materials, it is common practice to create samples mimicking historic materials (mock-ups of similar composition and manufacture technology) by subjecting them to processes of accelerated aging at elevated temperature, humidity and/or using light exposure. However, the relevance of the recommendations for exhibition and storage conditions based on such research, is often questioned in relation to both artificial aging and mock-up composition [2].

An alternative approach to the characterization of cultural heritage materials is to use small-scale mechanical testing techniques suitable for the direct examination of submillimeter samples. Instrumented indentation testing (IIT), also known as nanoindentation, is one such technique. Developed in the mid-1970s as an expansion of traditional hardness tests, it has become a well-established technique for characterizing relatively stiff materials such as ceramics and metals. A standard test consists of continuously measuring the load and displacement of an indenter as it is pressed into and withdrawn from the material. The load-displacement data contain an abundance of information about the tested material. The standard test provides the Young's modulus and hardness of the sample, but when more complex measuring schema are employed, its complex modulus, yield stress, creep 
and fracture toughness can also be characterized [3,4]. Nanoindentation was originally designed for the analysis of homogenous and nonviscous media, but over past decades new experimental protocols and methods of data analysis extended its applicability to a diverse range of materials. The versatility of this technique with respect to the determination of various material properties and accommodation of small sample sizes fills a needed role in the cultural heritage field.

In the next section of this review, nanoindentation studies of artists' paints performed over the last two decades are briefly presented. They are not introduced chronologically, but rather grouped to highlight various research questions and approaches adopted by researchers interested in analyzing paint media. The specificity of nanoindentation for such media is discussed in more detail in the following sections, which describe the nanoindentation technique itself, sample preparation, instrument calibration and the interpretation of results for free film and cross-sectional samples. Experimental methods and approaches are illustrated by results obtained at the Getty Conservation Institute or those already published elsewhere by other researchers.

\section{Nanoindentation Studies of Artists' Paints over the Last Two Decades}

In the last 20 years numerous research groups have used nanoindentation for the characterization of artists' paint media. The very first article discussing the results of nanoindentation for artists' paints was published by Chiantore and Scalone in 2007 [5]. They used nanoindentation to measure mechanical changes of modern acrylic paints artificially aged by exposure to UV light. Although the authors did not present quantitative analysis of the obtained data, they successfully demonstrated the sensitivity of nanoindentation to surface alternations resulting from accelerating aging.

Subsequent nanoindentation studies focused on quantitative evaluation of material properties through the use of model paint systems to explore and optimize the potential of the measuring technique. The storage moduli $\left(E_{\mathrm{r}}{ }^{\prime}\right)$ and hardness $(H)$ of embedded, cross-sectional samples of various artists' and commercially-available nonartists' paints (including oil-based enamel, acrylic, and alkyd paints) were measured by Wright et al. [6] Their study demonstrated the feasibility of the traditional Oliver and Pharr method [7] to analyze the mechanical properties of embedded paint samples. The authors also discussed the possible influence of pigments on the aging properties of paints, subsequently publishing a study analyzing the effects of zinc oxide, known as zinc white, on the curing and mechanical response of alkyd coatings [8]. Effects of zinc oxide pigment on the paint's properties were studied systematically at different concentrations over a wide range of time scales using three experimental techniques: dynamic mechanical analysis, quartz crystal rheometer and nanoindentation. The potential of using nanoindentation alongside other testing techniques for studying the mechanical properties of artists' paints was further explored in a recently published review article by Gwen dePolo et al. [9].

Nanoindentation was proven effective for evaluating mechanical changes of paint films following conservation treatments. Kaszowska et al. [10] analyzed the effect of consolidation of model wall paints (executed in three techniques: lime, lime casein and animal glue) with selected commercial synthetic binders. The experimental methodology applied in this work measured subtle differences resulting from various consolidation treatments but generally showed that they have a rather limited impact on the mechanical performance of the tested wall paints. The purpose-cast samples were also used for quantifying the effects of solvent exposure on a water-sensitive yellow ochre paint [11,12]. These authors reported an increase of paint stiffness after immersion in deionized water but an absence of changes following immersion in other tested solvents or following sponge cleaning with all tested solvents including water. These studies demonstrated how nanoindentation can help inform the selection of conservation materials and guide conservation treatments.

The most important feature of nanoindentation is its ability to measure small amounts of materials, which is critical to the analysis of minute samples taken from real art objects. 
Micro and nanoscale indentations have been applied to the characterization of samples recovered from seventeenth and nineteenth century oil paintings [13,14], as well as medieval distemper wall paints [15]. Such tests provide unique information unattainable by other techniques on how natural aging influences the mechanical properties of the measured materials. However, all three studies emphasize the difficulty in obtaining precise and representative mechanical properties due to high material heterogeneity and small sample size. They show that selection and preparation of samples are among the crucial issues when analyzing historic paints by nanoindentation.

Some fundamental problems related with how artists' paint samples are prepared for characterization of their mechanical properties were addressed in a series of articles prepared at the Getty Conservation Institute. The effect of infiltration of resin when embedding the paint sample [16], and the effect of indenting a finite-volume sample close to its edge [17], were analyzed experimentally. In both studies, dynamic indentation with a Berkovich indenter [18] was used to continuously monitor the complex stiffness as a function of penetration depth. The performed analyses resulted in the development of testing protocols dedicated to cross-sectional samples of paint that are small in volume, embedded in a material of dissimilar properties, and have a rough surface. The most recent work from this group proposes an original method of using a flat punch indenter to characterize soft and viscoelastic paints [19].

Dynamic indentation was also used by other researchers. In the previously mentioned work of Tiennot et al. [13], a testing protocol using a spherical probe, originally designed for biomechanical research on soft tissues, was adapted to analyze samples from a 17thcentury Rijksmuseum painting by Jan Baptiste Weenix. It was demonstrated that the developed measuring system can produce a two-dimensional map of modulus-similarly to that using atomic force microscopy - over a relatively large area of the sample. More recently, they used the same purpose-built system to analyze the impact of repeated relative humidity variations on the viscoelastic behavior of laboratory-prepared paint stratigraphies [20]. The use of a spherical indenter was also instrumental in the work of Giorgiutti-Dauphiné at al. [21]. They studied the role of substrate in the formation of craquelure patterns in paintings using a multilayered model system. A crack pattern observed in paintings was mimicked by drying dispersions of colloidal particles in a volatile solvent on a nonporous substrate. The nanoindentation results of the mimicked system showed that it is possible to deduce the elastic properties of the sublayer by measuring the length-scale of the stress relaxation dictated by the crack pattern. Although the measured system was substantially different from the original painting, the proposed method shows that the application of nanoindentation in heritage studies can provide unique information beyond quantifying hardness and modulus in very small scales.

Indentation studies of artists' paints carried out over the past two decades address a wide range of research questions. They also show that to take full advantage of the technique it is necessary to adapt it to the specific sizes and properties of historic and artists' paint samples.

\section{Instrumented Indentation Testing}

\subsection{Quasi-Static Method}

An indentation test consists of pressing an indenter of a well-defined geometry into the sample surface, with the resulting load-displacement curve serving as the 'mechanical fingerprint' of the material. The schematic representation of the process is presented in Figure 1. 

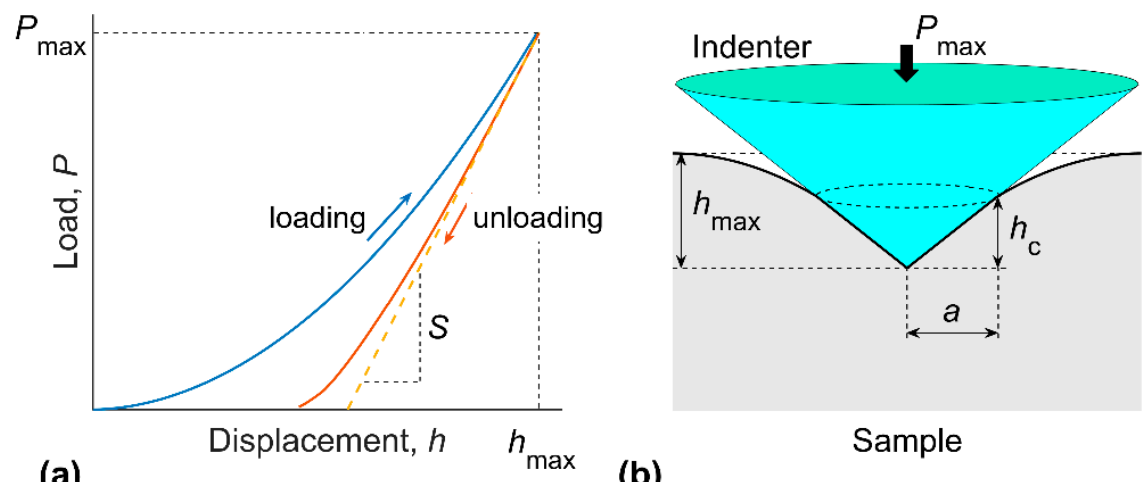

Figure 1. (a) Load versus displacement curve and (b) deformation of a sample upon indentation by a conical indenter.

While both the loading and unloading responses are recorded, the latter is used to determine the contact stiffness $(S)$ of the tested material using a curve fitting procedure from the widely accepted Oliver-Pharr method $[7,18]$. The depth of the material in contact with the indenter, or the contact depth $\left(h_{\mathrm{c}}\right)$, may be predicted from the displacement $\left(h_{\max }\right)$, load $\left(P_{\max }\right)$ and contact stiffness at the onset of unloading as $h_{\mathrm{c}}=h_{\max }-0.75 P_{\max } / S$ [18]. The projected area of the contact $\left(A_{\mathrm{p}}\right)$ is obtained as a function of contact depth based on knowledge of the indenter geometry $[7,18]$. The hardness $(\mathrm{H})$ is defined as the ratio of the maximum load to the projected contact area, or $H=P_{\max } / A_{\mathrm{p}}$. Using Sneddon's solution [22], the reduced elastic modulus $\left(E_{\mathrm{r}}\right)$ is given as the contact stiffness normalized to the lateral dimension of the contact, or $E_{\mathrm{r}}=S /(2 \beta a)$, where $a\left(=\sqrt{A_{\mathrm{p}} / \pi}\right)$ is the equivalent contact radius of a pyramidal indenter, and $\beta$ is a geometric constant of the indenter on the order of unity $[18,23]$. In the case of an axisymmetric indenter geometry such as a cone, sphere or flat-ended cylindrical punch, $\beta$ equals unity. The obtained $E_{\mathrm{r}}$ value is related to the Young's modulus $(E)$ and Poisson's ratio $(v)$ of the tested material and those of the indenter itself (indicated by the subscript i) through the relation $1 / E_{\mathrm{r}}=\left(1-v^{2}\right) / E+\left(1-v_{\mathrm{i}}^{2}\right) / E_{\mathrm{i}}$. Practically, since the Young's modulus of the indenter (the probe itself) is several orders of magnitude higher than that of the tested paint, the last term in the above equation can be omitted so that it reduces to $E_{\mathrm{r}}=E /\left(1-v^{2}\right)$. Determination of the Young's modulus is limited by the lack of knowledge about the Poisson ratio of the tested material; therefore, the reduced modulus is often reported instead.

For time-dependent materials (such as artists' paints), it is possible to visualize their creep or stress-relaxation behavior by adding a pause between the loading and unloading phases. Creep is determined by measuring the deformation under a constant load (in a loadcontrolled experiment), while stress-relaxation examines change of load under a constant displacement (in a depth-controlled experiment). While virtually any nanoindentation instrument is capable of measuring the time-course creep displacement, its precise measurement requires accounting for thermal drift. One way of ensuring correct measurements of the indenter tip position over an extended period of time is to use a reference probe placed in close proximity to the tested surface. Such a measuring system [24] was proven to be particularly effective for characterizing the creep behavior of an acrylic paint [16]. However, since creep parameters determined by nanoindentation do not directly correspond to those measured in uniaxial testing, its usefulness may be limited for comparative purposes within similar materials or test systems.

\subsection{Dynamic Method}

The continuous stiffness measurement (CSM) technique [18,25-28] is an effective method for dynamically characterizing the indentation response of a time-dependent material. This technique measures the stiffness dynamically, typically during the loading phase of the sample, by imposing small-amplitude sinusoidal oscillations on the monotonically increasing load. In the case of a time-independent material, for which the oscillations in 
displacement and load are in phase with each other, the dynamic stiffness $\left(S^{*}\right)$ reduces to the ratio of the load amplitude $(\Delta P)$ to the displacement amplitude $(\Delta h)$, or $S^{*}=\Delta P / \Delta h$. In the case of a viscoelastic material, for which the displacement lags behind the load by a phase angle $\delta$, the dynamic stiffness consists of in-phase and out-of-phase components expressed as $S^{\prime}=(\Delta P / \Delta h) \cos \delta$ and $S^{\prime \prime}=(\Delta P / \Delta h) \sin \delta$, respectively. $S^{\prime}$ is termed the storage stiffness that describes the stiffness of the contact, while $S^{\prime \prime}$ is the loss stiffness that equals the damping of the contact multiplied by the angular frequency of the dynamic oscillation. Based on Sneddon's solution together with the elastic-viscoelastic correspondence principle, normalizing the storage and loss stiffnesses to the contact dimension $(2 \beta a)$ yields the reduced storage and loss moduli as $E_{\mathrm{r}}^{\prime}=S^{\prime} /(2 \beta a)$ and $E_{\mathrm{r}}^{\prime \prime}=S^{\prime \prime} /(2 \beta a)$, respectively. The loss tangent (or $\tan \delta$ ), defined as the ratio of loss modulus to storage modulus ( $\left.\tan \delta=E_{\mathrm{r}}^{\prime \prime} / E_{\mathrm{r}}^{\prime}\right)$, describes the viscous state of the material; $\tan \delta$ ranges from zero for an ideal elastic solid to infinity for an ideal liquid. The dynamic indentation technique may thus be regarded as comparable to dynamic mechanical analysis (DMA). Considering the definitions of the storage and loss moduli, it is apparent that these measurements are dependent on the accuracy of determining the (equivalent) contact radius $a$, whereas $\tan \delta$ remains unaffected.

An example of indentation testing in the CSM mode using a pyramidal (Berkovich) indenter on a $0.2 \mathrm{~mm}$-thick $\mathrm{TiO}_{2}$ acrylic paint film glued onto a glass substrate is presented in Figure 2. Dynamic force oscillations are applied during the loading phase of the sample with an exponentially increasing force. Using such an exponential loading scheme with a pyramidal (Berkovich) indenter, a constant strain rate of the sample can be maintained for a homogeneous material [29]. Such a test is equivalent to a uniaxial tensile or compression experiment with a linearly varying displacement, thereby keeping the strain rate constant during the test. It is practically impossible to maintain a constant strain rate during the loading phase with any indenter geometry other than pyramidal, or during the load-hold or unloading phase with any geometry.

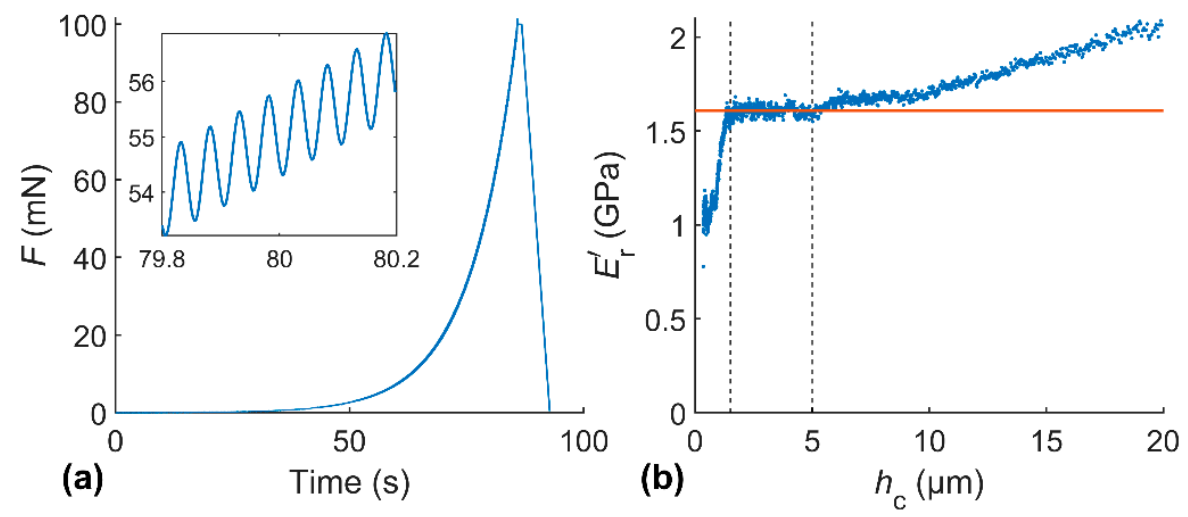

Figure 2. (a) CSM load function applied to a $0.2 \mathrm{~mm}$-thick $\mathrm{TiO}_{2}$ acrylic paint film on a glass substrate, and (b) the resulting reduced storage modulus versus contact depth, showing the effects of surface roughness and substrate stiffness below $\sim 1.5 \mu \mathrm{m}$ and above $\sim 5 \mu \mathrm{m}$, respectively.

Figure $2 \mathrm{~b}$ demonstrates an immediate advantage of the dynamic test and its ability to characterize material properties (reduced storage modulus in this case) at different depths from the surface. If the material is homogeneous, one can define the depth range at which $E_{\mathrm{r}}^{\prime}$ is affected by the roughness of the sample surface (at small indentation depths) or the substantially greater stiffness of the underlying substrate (at large indentation depths). As a result, dynamic indentation offers the user a means of identifying the valid depth range of indentation at which systematic errors due to the finite size of the sample and its preparation method can be minimized. 


\subsection{Probe Geometries}

Various probe materials and geometries can be employed for indentation tests. Manufacturing precision and desired high hardness and stiffness favor diamond as the material of choice; however, other materials, such as sapphire, hardened steel and tungsten, are also used. These less stiff materials are adequate for measuring historic and artists' paints since their rigidity is many orders of magnitude greater than those of paints, making their compliance negligible when analyzing the test results. With respect to probe shapes, there are three commonly used geometries offering radically different opportunities: pyramid, flat-ended cylindrical punch, and sphere.

Sharp indenters are frequently used in nanoindentation. The simple axial symmetry makes a conical geometry attractive from a modelling standpoint, but the difficulty in manufacturing conical diamonds prevents the use of such indenters in practice. In contrast, pyramidal probes, of which the Berkovich indenter is the most popular, are relatively straightforward to manufacture and used in many practical applications. The well-defined three-sided pyramidal geometry allows one to subject the material to a constant strain during the loading phase [30]. This is because the representative strain exerted in the material scales with the tangent of the complimentary angle $(\alpha)$ between the sample surface and the indenter flank [30]. As previously stated in Section 3.2, the strain rate during sharp indentation can be held constant for a homogeneous material with the use of a specific loading scheme [29]. Indentation with a diamond Berkovich indenter typically induces plastic deformation of the sample as the imposed strain beneath the indenter is sufficient to cause yielding of many materials. The main disadvantage of using a sharp indenter is the difficulty in defining the contact depth when material 'piles up' around the indenter as a consequence of plastic flow outside the contact perimeter. Incorrect evaluation of the contact depth, and hence of the projected contact area and equivalent contact radius, induces errors on the nanoindentation-derived hardness and modulus [31].

A flat-punch indenter has the form of a vertical cylindrical column with a flat circular base. From the moment of full contact between the circular base of the indenter and the material, the contact area remains well-defined and constant, eliminating the uncertainty of defining the contact area/radius encountered in sharp indentation. The main disadvantage of flat-punch indentation is its relatively large contact radius, which makes the test results more sensitive to the stiffness of the surrounding substrate material [19]. Further, unlike in the case of sharp indentation, the strain field beneath the flat-punch tip changes with indentation depth in a complex manner. Deep indentation of a soft and viscoelastic film beyond the limit of linear viscoelasticity results in an erroneous modulus measurement due to the lack of a universally applicable solution for deducing the reduced modulus in the nonlinear viscoelastic deformation regime.

A spherical indenter offers another means of probing a material. Unlike in sharp indentation, the strain imposed by a spherical indenter at a limited contact force can be kept small enough to maintain the sample deformation in the elastic regime. With an increasing indentation load, the average strain (dictated by $\tan \alpha$ ) increases progressively with indentation depth, resulting in a transition from elastic to plastic deformation. Defining the exact point of this transition can enable the determination of the yield stress of the tested material $[30,32,33]$, although its accuracy may be hindered by the fact that manufacturing perfectly spherical probes at the micrometer scale is inherently difficult. Spherical indentation, when taken well beyond the elastic limit, can suffer the same problem of incorrectly evaluating the contact area as in sharp indentation. Since the 'pile-up' height in spherical indentation is not scalable with the contact dimension, the percentage error in modulus estimation may increase with indentation depth [18].

Table 1 summarizes the benefits and limitations of the described probes. It also compiles the types of probes and the measuring techniques used in the studies described in Section 2. 
Table 1. Information about probe types and measuring schema applied in nanoindentation studies of artists' paints (RED—studies of original historic materials; BLUE—studies of mock-ups and model systems; GREEN—treatment/aging studies on mock-ups).

\begin{tabular}{|c|c|c|c|c|c|}
\hline & \multirow[t]{2}{*}{ Specific Geometry } & \multirow[t]{2}{*}{ Benefits } & \multirow[t]{2}{*}{ Limitations } & \multicolumn{2}{|c|}{$\begin{array}{l}\text { Studies on Mock-Ups or Original } \\
\text { Historic/Artists' Paints }\end{array}$} \\
\hline & & & & Quasi-Static Method & Dynamic Method \\
\hline Sharp & $\begin{array}{c}\text { Three-sided } \\
\text { pyramid- } \\
\text { Berkovich }\end{array}$ & $\begin{array}{l}\text { Manufacturable with } \\
\text { high precision; } \\
\text { constant strain and } \\
\text { strain rate during } \\
\text { the test }\end{array}$ & $\begin{array}{l}\text { Underestimated } \\
\text { contact radius due to } \\
\text { 'pile-up', hence over- } \\
\text { estimated modulus }\end{array}$ & $\begin{array}{c}{[5]} \\
{[2]} \\
{[6]} \\
{[10]} \\
{[16]} \\
{[8]} \\
{[15]}\end{array}$ & $\begin{array}{c}{[14]} \\
{[2]} \\
{[11]} \\
{[17]} \\
{[16]} \\
{[12]}\end{array}$ \\
\hline Flat punch & $\begin{array}{l}\text { Column with flat } \\
\text { circular base }\end{array}$ & $\begin{array}{l}\text { Well defined and } \\
\text { constant contact } \\
\text { radius when in } \\
\text { full contact }\end{array}$ & $\begin{array}{l}\text { Small deformation } \\
\text { assumption; } \\
\text { pronounced } \\
\text { substrate effect }\end{array}$ & & [19] \\
\hline Spherical & Sphere & $\begin{array}{c}\text { Solely elastic } \\
\text { deformation at initial } \\
\text { contact at a } \\
\text { small load }\end{array}$ & $\begin{array}{l}\text { Non-scalable strain } \\
\text { field, hence 'pile-up' } \\
\text { with depth }\end{array}$ & & $\begin{array}{l}{[21]} \\
{[13]} \\
{[20]}\end{array}$ \\
\hline
\end{tabular}

\section{Characterization of Artists' Paints}

Paint is a heterogeneous blend of pigments, binders, and additives, which affect its workability and drying properties. Initially liquid-like paint changes into a solid-like film through drying or crosslinking process. This change takes place over weeks, months and years, and is dependent on the paint composition and ambient environmental conditions, resulting in a dramatic increase of paint stiffness [34-37]. Paints applied successively by brush on a substrate form a multilayered structure. The thicknesses of individual layers in old masters' paintings can be as thin as 5-50 $\mu \mathrm{m}$ [38]. On the other hand, the ground layers and impastos found in modern and contemporary paintings can be as thick as a few millimeters. The mechanical properties of individual paint layers are affected by the surrounding materials and stresses that are built up in the structure during the drying process [39-42]. When measuring the mechanical properties of paints at the microscale, it is important to account for several factors, including the heterogeneity of the film as dictated by the size(s) and distribution of the pigment particles, the overall drying time, and effects from the surrounding materials and environmental conditions (temperature and relative humidity).

\subsection{Free Film Paint Samples}

Due to their severely limited volumes, historic samples are generally not available for mechanical testing in the form of a film. However, purposely cast, and artificially or naturally aged materials are often used as mock-ups of historic paints. These proxy samples can be used to study changes in the mechanical properties of a paint resulting from a particular composition [8], aging process [5,9], or conservation treatment [10,12]. Paints can be cast directly on a rigid substrate (e.g., glass plate) or transferred to such a substrate after drying. Such samples are referred to as free films in this article (as opposed to embedded cross-sections). Sample preparation must focus on choosing not only the formulation of the paint to be reproduced, but also the thickness of the paint film, as the curing process can impact the distribution of mechanical properties.

\subsubsection{Testing Area}

From the viewpoint of nanoindentation testing, the main advantage of a free film sample is its large surface area that enables multiple indentation tests to be performed. 
Since many paints are strongly heterogeneous, it is advantageous to perform tests at a number of locations on the sample surface, and to analyze the results statistically. Such nanoindentation tests may show how strongly the presence of pigment particles (which may have different stiffnesses and sizes ranging from tenths of nanometers to tenths of micrometers) influences the local mechanical properties and whether the average properties of the constituent materials can be deduced statistically. The limitation of nanoindentation in testing highly pigmented, granular, and porous laboratory prepared gesso and historic distemper paint has been explored [15]. Multiple quasi-static indentation tests successfully informed the average properties of the laboratory sample, but not of the significantly more porous historic one. The difference in porosity of these samples was confirmed by $\mathrm{X}$ ray micro-CT scans.

\subsubsection{Depth of Indentation}

The mechanical properties of many paint films can be established, despite the presence of pigment, by increasing the depth of indentation. In practice, large indentation depths may be needed to accommodate the inherent roughness of the paint surfaces. The rule of thumb dictates that the depth of indentation be at least $\sim 20$ times the roughness depth, which amounts to an indentation depth of at least few microns for a typical paint surface. The obvious limitation of indentation depth is the maximum limit of force achievable by a nanoindenter. However, before this instrumental limit is reached, the measured mechanical response of the paint film sample may be affected by the presence of the substrate material to which it is adhered. Though a common approach to avoiding the substrate effect is to limit indentation depth to less than $10 \%$ of the film thickness, this may only help avoid large errors in establishing hardness and does not always guarantee the obtained modulus to be accurate. As clearly visible in Figure 2, the dynamic measurement of the $0.2 \mathrm{~mm}$ thick acrylic paint reaches the onset of the substrate effect on $E_{\mathrm{r}}^{\prime}$ at the contact depth of $\sim 5 \mu \mathrm{m}$ ( $2.5 \%$ of the film thickness). The extent to which the substrate affects the measured response of the paint film depends on the depth of indentation relative to the film thickness, as well as the modulus mismatch ratio between the film and the substrate. Since the modulus of the paint film is unknown a priori, this ratio is also unknown at the stage of defining the experimental parameters.

The only practical way to establish the depth range within which the mechanical response of a given paint film is free from the substrate effect is through an examination of the measured modulus as a function of depth. This can be achieved either by the multiple partial unloading indentation technique [32] or the CSM technique. In the example of the $0.2 \mathrm{~mm}$-thick acrylic paint given in Figure 2, the reduced modulus remains constant from 1.5 to $5 \mu \mathrm{m}$ in contact depth, indicating that the measurement in this range is unaffected either by the surface roughness or the substrate stiffness.

In the case of sharp indentation, this information can also be obtained from the measured stiffness $\left(S^{\prime}\right)$ plotted as a function of contact depth $\left(h_{\mathrm{c}}\right)$. For a pyramidal (or conical) indenter, the equivalent contact radius (a) increases with the contact depth; hence, the measured stiffness is a linear function of contact depth for a homogeneous material. The storage stiffness versus contact depth of the $0.2 \mathrm{~mm}$-thick acrylic paint, plotted in Figure 3, indeed deviates from linearity at small $(<\sim 1.5 \mu \mathrm{m})$ and large $(>\sim 5 \mu \mathrm{m})$ depths due to the effects of surface roughness and substrate stiffness, respectively. 

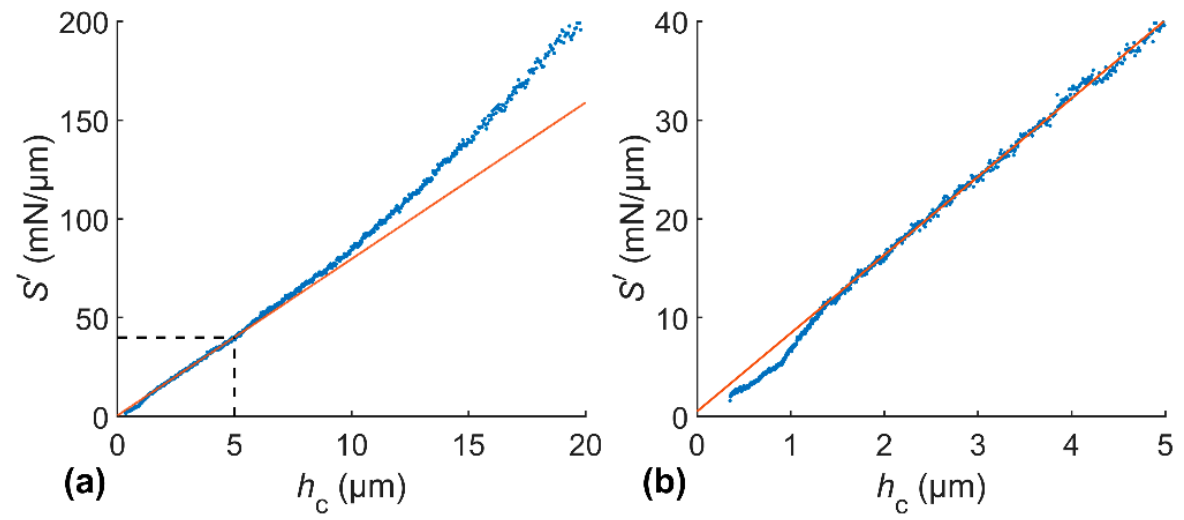

Figure 3. Storage stiffness versus contact depth of a $0.2 \mathrm{~mm}$-thick $\mathrm{TiO}_{2}$ acrylic paint film on a glass substrate (other results on the same sample shown in Figure 2), deviating from linearity indicated by the red line (a) above $\sim 5 \mu \mathrm{m}$ caused by the stiffness of the underlying glass substrate and (b) below $\sim 1.5 \mu \mathrm{m}$ due to the surface roughness of the film.

The stiffness versus contact depth plot (Figure 3) also allows one to systematically and consistently establish the 'effective' contact point of the sharp indenter with a rough sample. In instrumented indentation, the initial contact point is typically defined as the instance at which the probe detects an extremely small amount of force or stiffness above a selected threshold, which is a result of the probe physically contacting the sample surface. For samples with a rough surface, however, such a procedure can result in an over- or under-estimation of the 'effective' indentation depth. To illustrate such possibilities, the surface profile of a laboratory prepared film of $\mathrm{TiO}_{2}$ acrylic paint is presented in Figure 4a. The analyzed surface profile, shown in Figure $4 \mathrm{~b}$, was taken from a smooth area free from surface defects or irregularities. Such areas are commonly selected as preferred locations for nanoindentation testing. Across the $10 \mu \mathrm{m}$ length of the scanned surface profile, the maximum deviation from the average profile (shown as the dashed line) is $\pm 0.1 \mu \mathrm{m}$. This difference corresponds to $\pm 10 \%$ of the effective indentation depth at an indenter displacement of $1 \mu \mathrm{m}$ below the dashed line. If we assume that the sample is contacted by an ideally sharp indenter in a solely elastic manner, the theoretical contact depth in the absence of surface roughness is given by $h_{\mathrm{c}}=(2 / \pi) h_{\max } \approx 0.64 \mu \mathrm{m}$. The $\pm 10 \%$ error in $h_{\max }$ thus causes the Oliver-Pharr method $\left(h_{\mathrm{c}}=h_{\max }-0.75 P_{\max } / S\right)$ to over or under-estimate $h_{\mathrm{c}}$ by $0.1 \mu \mathrm{m}$, which amounts to $\pm 16 \%$ error in contact depth, hence $-14 \%$ or $+19 \%$ error in modulus, respectively. The percentage error in modulus decreases progressively with indentation depth and falls below $3 \%$ in magnitude at depths above $5 \mu \mathrm{m}$ for the same simulation. Thus, theoretically, uncertainty in establishing the contact depth can be minimized by increasing the depth of indentation. For thin paint films with a rough surface, however, there is a limit as to the depth of an indentation without triggering the substrate effect.

The effective contact point in sharp indentation can be established by fitting a linear function to the stiffness versus contact depth curve in the range not affected by the surface roughness or the substrate stiffness and extrapolating it to zero stiffness (Figure 3). This method, however, is only applicable for a homogenous material for which a linear relationship holds between the stiffness and the contact depth in the above specified range. 


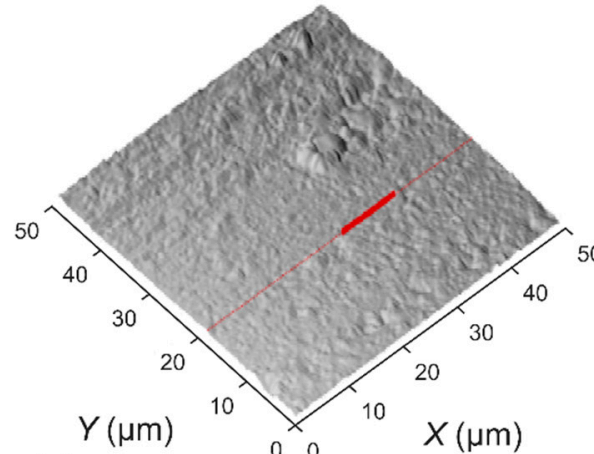

(a)

Figure 4. (a) AFM scan of a laboratory prepared $\mathrm{TiO}_{2}$ acrylic paint, and (b) two-dimensional surface profile of the paint along the thick segment of the red line in (a) contacted by an indenter with a half-included angle of $70.3^{\circ}$, showing uncertainty in defining the contact depth on a rough surface. Note that the aspect ratio of the indenter (indicated by the blue triangles) is correctly scaled with the surface profile.

\subsubsection{Contact between Indenter and Sample}

Surface roughness is not the only source of error in determining the contact depth $\left(h_{\mathrm{c}}\right)$, and, consequently, the projected contact area $\left(A_{\mathrm{p}}\right)$ and the equivalent contact radius $\left(a=\sqrt{A_{\mathrm{p}} / \pi}\right)$. The Oliver-Pharr method calculates the contact depth with a pyramidal or conical indenter as $h_{\mathrm{c}}=h_{\max }-0.75 P_{\max } / S$, assuming that the deformation of the sample occurs solely in elastic manner [18]. However, many materials deform both elastically and plastically upon indentation by a diamond Berkovich indenter. Fortunately, the contact equation works reasonably well for an elasto-plastic solid that work-hardens appreciably during the indentation test [31]. For a non-work-hardening solid, however, the equation does not work well as the material 'piles up' around the indenter.

The surface profile of a material with 'pile-up', shown schematically in Figure 5b for a conical indenter geometry, is a consequence of the plastic flow of the material outside the contact perimeter. Since the contact equation assumes the absence of 'pile-up' (Figure 5a), it is bound to underestimate the contact depth in the presence of 'pile-up' [18,31]. As a result, the nanoindentation-derived modulus $(\propto 1 / a)$ and hardness $\left(\propto 1 / A_{\mathrm{p}}\right)$ of the specimen are overestimated. The extent to which a material 'piles up' can be pronounced when testing a thin film constrained by a much harder and stiffer substrate [43]. The occurrence of 'pile-up' can be confirmed by microscopic (optical or AFM) observation of the indentation imprint. If 'pile-up' has occurred during the test with a pyramidal indenter, the sides of the residual impression appear bowed-out, whereas excessive 'sinking-in' results in the sides of the impression bowing-in, as shown in Figure 6a. The contact equation works well for the residual imprints on the left and in the middle of Figure 6a but underestimates the contact depth for the imprint on the right. Theoretically, the Oliver-Pharr method can also overestimate the contact depth in the presence of excessive 'sink-in' [43]. Such a situation may occur while testing a hard film on a soft substrate, a rather unusual situation for paint samples.

Paints, especially those which are soft, can be notoriously prone to 'pile-up' issues. Since the properties of paints depend on temperature and relative humidity, the extent of 'pile-up' cannot be discussed without considering the environmental conditions during the test. The residual imprints of Winsor \& Newton ultramarine oil paint [11] with a Berkovich indenter, presented in Figure $6 \mathrm{~b}$, show the changing nature of the probe-material contact with increasing relative humidity. 'Sink-in' observed in the tests performed at $35 \% \mathrm{RH}$ is no longer present at $60 \% \mathrm{RH}$. At $70 \% \mathrm{RH}$ there is a clear manifestation of 'pileup', whereas at $83 \%$ RH the indenter leaves a shapeless imprint, indicative of the paint's liquid-like properties in this environment. Indentation testing of the ultramarine paint at high humidity, when analyzed using the Oliver-Pharr method, results in systematic 
overestimation of the paint modulus. The extent of this overestimation is difficult to assess without precise recording of the additional contact area created by 'pile-up'. This, however, is an extremely difficult, if not impossible, task for environmentally-softened paints as the indentation imprint may change with time, both with respect to shape and size, due to the pronounced time dependence of their mechanical properties. The change in the mechanical state of the paint due to increasing moisture levels (solid to liquid transition) can be monitored by measuring the change in reduced modulus. Nevertheless, the accuracy of the measured modulus is compromised due to more pronounced 'pile-up' at a higher relative humidity. The environmental condition at which the transition occurs can be more precisely established by measuring the loss tangent, whose determination is independent of the contact area.

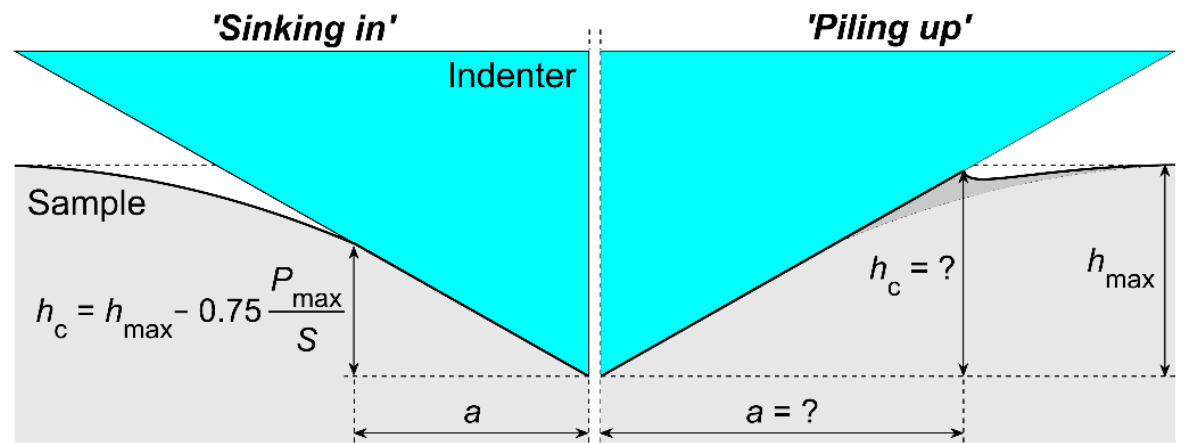

(a)

(b)

Figure 5. Surface profile upon sharp indentation of (a) an ideal elastic solid showing 'sink-in', and (b) elasto-plastic solid with little work-hardening characteristics showing 'pile-up' around the indenter.

(a)

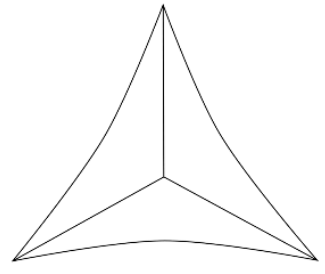

'Sink-in'

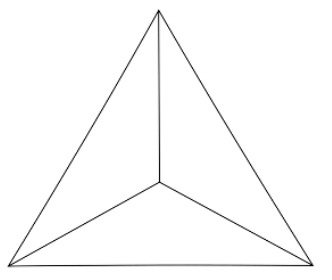

No 'sink-in' or 'pile-up'

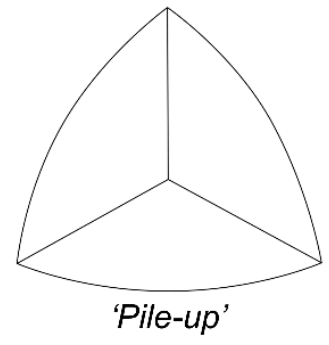

'Pile-up'

(b)

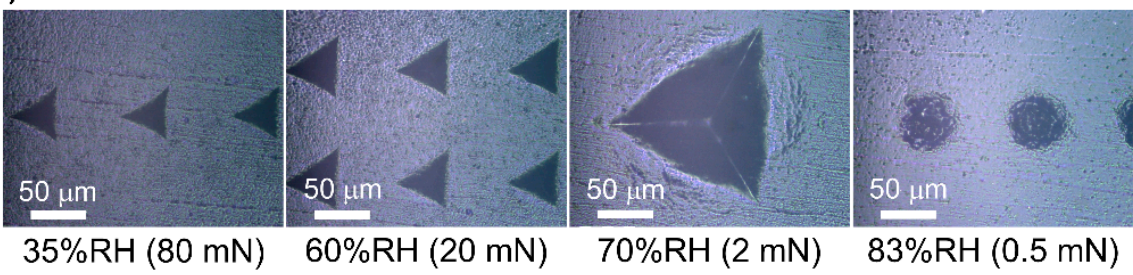

Figure 6. Residual imprints of a Berkovich indenter showing different degrees of 'sink-in' and 'pileup'; (a) schematic diagrams and (b) microscopic images of imprints recorded after indenting Winsor \& Newton ultramarine oil paint at different relative humidity levels (note differences in maximum load applied at different humidity levels).

Some modern paints remain in a soft and liquid-like state in ambient environmental condition [44,45]. Measuring the modulus for such materials requires a method that can establish the contact radius without reliance on the Oliver-Pharr contact equation. Dynamic indentation with a flat-punch indenter was proposed for characterizing extremely soft modern oil paints that exhibit exudates and drips in a standard climate-controlled museum environment [19]. The flat-ended cylindrical punch geometry provides a constant and well-defined contact radius. Due to its relatively large contact dimension, however, determining the modulus of a soft paint in thin-film form requires adequately accounting 
for the substrate effect. A solution of the substrate effect was derived numerically by Cao et al. for a wide range of contact radius to film thickness ratios [46]. However, unlike in the case of sharp indentation, the strain field beneath the flat-punch tip evolves with increasing depth in a complex manner. In order to apply Cao's solution, the dynamic mechanical properties of a soft paint film must be evaluated at a very small depth in the limit of linear viscoelasticity.

Precise knowledge of the contact dimension and the substrate effect are less important when changing properties, rather than their absolute values, are under investigation. For example, recent work by [20] dynamically assessed a multilayered mock-up paint system with a spherical indenter to determine the impact of repeated relative humidity variations on its viscoelastic behavior. The results of the study were not corrected for the substrate effect because comparisons of the relative contributions of the individual paint layers constituting the prepared sample was an intention of the study.

\subsubsection{Calibration and Cleaning of Sharp Indenter}

While 'pile-up' and roughness of the paint surface are two primary contributors to the discrepancy between the actual and theoretically predicted contact depths, improper calibration of the indenter shape as well as the contamination of the indenter tip can further impact this divergence. Typically, the shape of the Berkovich indenter is accurately defined with high precision and certified by the manufacturer. Nanoindentation defines the shape of the indenter with an area function, which describes the cross-sectional area of the indenter as a function of the distance from the apex. Such a function is routinely obtained using a calibration procedure developed by Oliver and Pharr [7,18], which involves indenting a reference material with a known Young's modulus and Poisson's ratio to a range of depths. The most commonly used reference material is fused silica. The area function derived from this calibration procedure allows one to define the exact shape of the indenter that may be imperfect or worn at extremely small depths. However, due to the high stiffness of fused silica (Young's modulus of $\sim 72 \mathrm{GPa}$ ), an indentation test with a maximum force available with most commercial nanoindentation instruments results in a contact depth no greater than $1 \mu \mathrm{m}$ (e.g., a maximum force of $100 \mathrm{mN}$ results in a contact depth with fused silica of $\sim 0.7 \mu \mathrm{m})$. To test a realistic paint sample with a considerably rough surface, it is necessary to define the indenter shape at contact depths well beyond this instrumental limit.

The three-dimensional shape of a pyramidal Berkovich indenter may be translated into an analogous two-dimensional conical indenter. This involves equating the triangularshaped cross-section of the indenter at a given contact depth to a circle, and regarding the radius of the circle $\left(a=\sqrt{A_{\mathrm{p}} / \pi}\right)$ as the equivalent contact radius of the indenter at that contact depth. An example of the two-dimensional shape function of a Berkovich indenter is given in Figure 7a. The equivalent contact radius $(a)$ versus contact depth $\left(h_{\mathrm{c}}\right)$ curve is fitted with a polynomial function in the form $a=C_{1} h_{\mathrm{c}}+C_{2} h_{\mathrm{c}}^{1 / 2}$, where the first term describes the global pyramidal geometry of the indenter and the second term accounts for the rounding of the indenter tip. Such a function may be used to extrapolate the indenter shape, but only slightly beyond the contact depth range of calibration. When it is necessary to extrapolate the shape function far beyond the existing data, it may be more appropriate to use a linear function fitted to the linear portion of the data (i.e., $h_{\mathrm{c}} \geq 200 \mathrm{~nm}$ for this example, Figure $7 \mathrm{~b}$ ).

To assess if the fitted calibration function adequately describes the shape of the indenter at several microns in contact depth, indentations may be performed on a bulk homogeneous material that is much more compliant than fused silica. In the GCI laboratories, a $9.5 \mathrm{~mm}$ thick polycarbonate sheet (Lexan) is used routinely for this task, with a resulting contact depth of $\sim 5 \mu \mathrm{m}$ at $100 \mathrm{mN}$ (depending on the loading condition used). At this depth, the substrate effect is nonexistent, and the reduced storage modulus measured with the CSM technique should remain constant over the entire depth range (excluding data collected at shallow depths influenced by surface roughness and tip imperfections). The results presented in Figure 7c show that the calibration function based on the applied procedure 
successfully extrapolates the indenter shape over the depth range necessary for testing realistic paint samples.
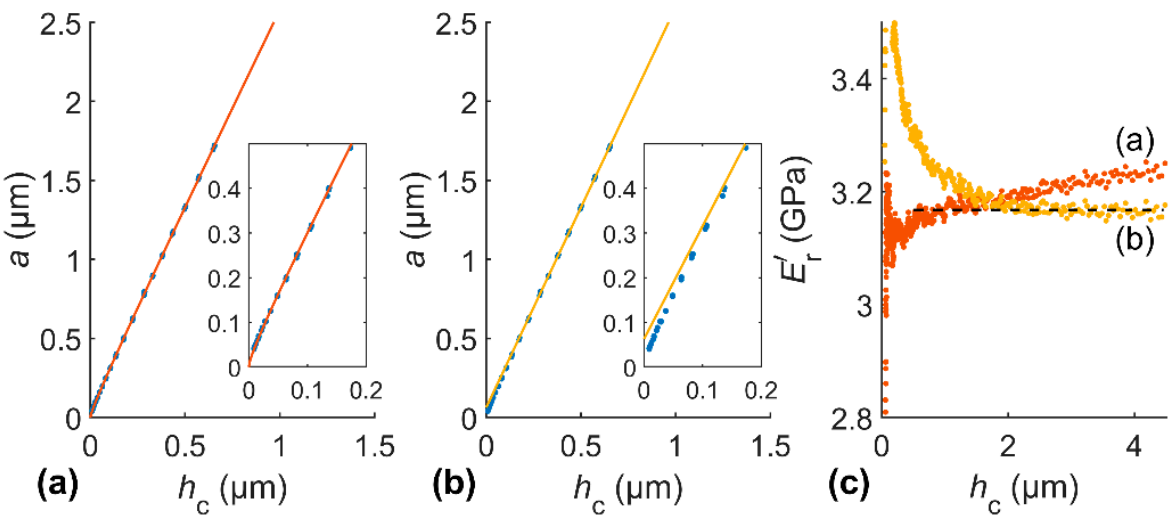

Figure 7. Indenter shape function (a) $a=C_{1} h_{\mathrm{c}}+C_{2} h_{\mathrm{c}}^{1 / 2}$, fitted to the entire contact depth range of fused silica data, (b) $a=C_{3} h_{\mathrm{c}}$, fitted to the range above $200 \mathrm{~nm}$, and (c) reduced storage modulus versus contact depth of polycarbonate obtained using the respective shape functions in corresponding colors, where the dashed line shows the actual modulus of the material.

Indentation on polycarbonate also serves as an effective method for cleaning the indenter. Soft and viscous paints readily contaminate the indenter tip, leading to systematic errors in successive measurements. Contamination of a Berkovich tip after a series of indentations on three Winsor \& Newton oil paints (Ultramarine Blue, yellow Ochre and Cadmium Yellow) is documented using scanning electron microscopy (SEM) in Figure 8a. The same indenter after manual cleaning with a cotton swab soaked in isopropanol is shown in Figure 8b.
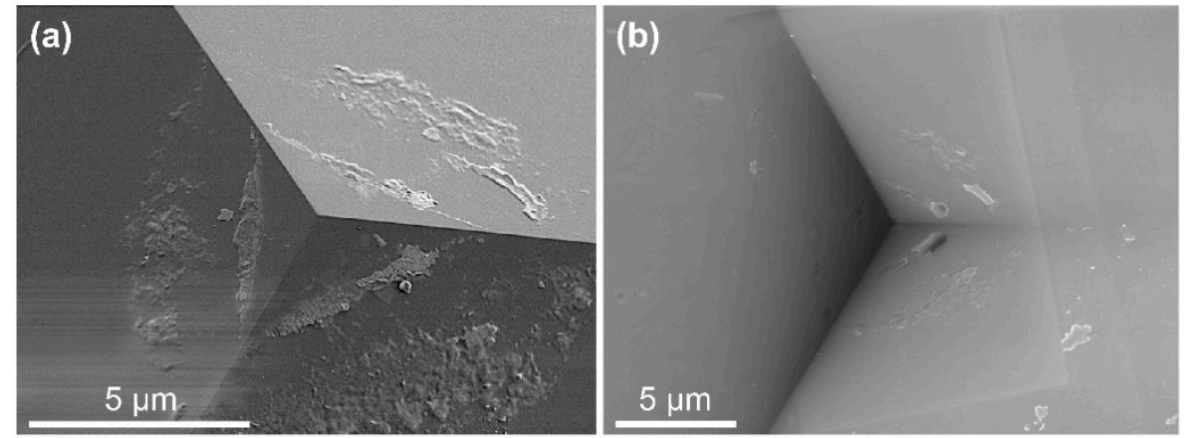

Figure 8. SEM images of a Berkovich tip contaminated with oil paints (a) before and (b) after manual cleaning with cotton swab soaked in isopropanol.

These SEM images show that the applied cleaning method removed much of the paint residue from the indenter tip. However, the effectiveness of the manual cleaning method can vary, and it is impractical to confirm the cleanliness of the indenter frequently with SEM. A simple and effective technique to clean the indenter tip involves indenting polycarbonate with the maximum available force. During this process, friction at the interface with the polycarbonate sample remove paint residue adhered to the diamond indenter tip. Whichever method is chosen for cleaning the tip, its effectiveness can be verified by indentation in polycarbonate. This process can be repeated until the measured stiffness versus depth curve shows a profile identical to that of the same tip in a clean condition. An example of such a procedure is presented in Figure 9 for a Berkovich indenter contaminated with Liquitex acrylic gesso. When the risk of tip contamination is high, it is good practice to indent polycarbonate (or another material used for cleaning) prior to performing any new test of a sample. 


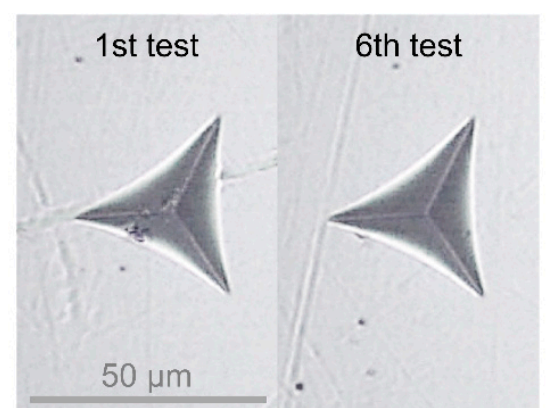

(a)

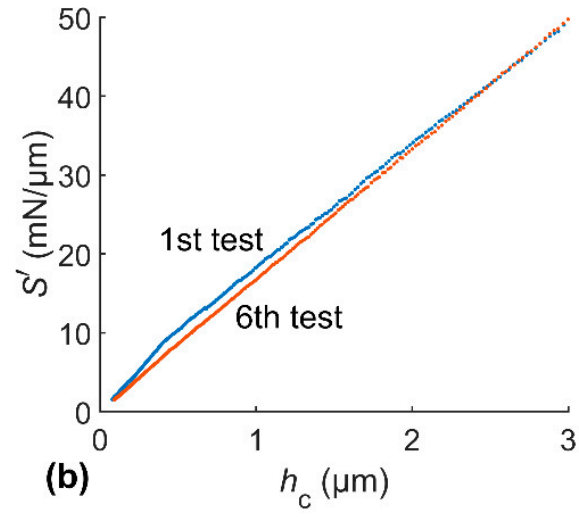

(b)

Figure 9. Cleaning of a Berkovich indenter contaminated with Liquitex acrylic gesso by successive indentions into polycarbonate; (a) the imprint of the first test (left) showing the paint residue on the sample surface and that of the sixth test (right) showing no signs of contamination, and (b) comparison of stiffness versus contact depth for the first and sixth tests.

\subsection{Cross-Sectional Samples}

Nanoindentation can be employed to determine the mechanical properties of submillimeter cross-sectional samples obtained from actual paintings. In principle, materials sampled during routine documentation of even the most valuable works of art can be characterized and compared with laboratory-prepared mock-ups. Testing cross-sectional paint samples (which are common in the field of cultural heritage conservation) can also inform the distribution of mechanical properties across a typical collection of a certain age and region and how natural aging influences the properties of these paints. Nanoindentation of cross-sectional samples has similar problems to the testing of free film samples, as discussed in Sections 4.1.2-4.1.4. In addition, the successful adaptation of nanoindentation methods to cross-sectional paint samples requires understanding how sample preparation may influence the measured results. Embedding and mounting practices can alter the properties of the paint samples and render prepared cross-sections unsuitable for nanoindentation testing.

\subsubsection{Preparation of Sample Surface}

In heritage conservation, the main purpose of preparing cross-sectional samples is to optically study paint stratigraphy. This requires vertically embedding a sliver of paint in a transparent medium (resin) for mechanical stabilization, and polishing its surface [47]. Unlike for free film samples, the surface of a cross-sectional sample, held securely in an embedding material, can be prepared in a more controlled manner for indentation testing. Till now, dry hand polishing of the surface was the sole method of surface preparation when indenting cross-sections $[2,13,14,16,17]$. The process can be carefully controlled using progressive grades of Micro-Mesh sanding sheets. Nanoindentation measurements are conducted after applying various pressures during polishing to check whether the process has not altered the mechanical properties of the surface. Other potential methods of cross-sectional sample surface preparation, such as microtoming and focused ion beam milling, are more difficult to apply. The former is hindered by specific geometry and size requirements, while the latter risks changing the surface properties due to exposure to the high intensity ion beam. Paint cross-sections prepared with these alternate methods have yet to be studied by nanoindentation.

\subsubsection{Indentation near Sample Edge}

Two main issues associated with testing embedded cross-sectional samples are infiltration of the embedding medium into the sample and edge effect. Figure 10 shows the Berkovich indentation imprints on the cross-sectional surfaces of a $0.26 \mathrm{~mm}$-thick $\mathrm{TiO}_{2}$ acrylic paint film (generated with $9 \mathrm{mN}$ maximum load) and its surrounding Technovit ${ }^{\circledR}$ 
2000 LC liquid as the embedding resin material (generated with $100 \mathrm{mN}$ maximum load). While all indents on the are identical in both size and shape, an indent on the paint near the sample-resin interface is smaller. This is attributed to the liquid resin infiltrating the paint during the embedding process, thereby increasing the measured hardness of the paint sample near the interface in cured condition.

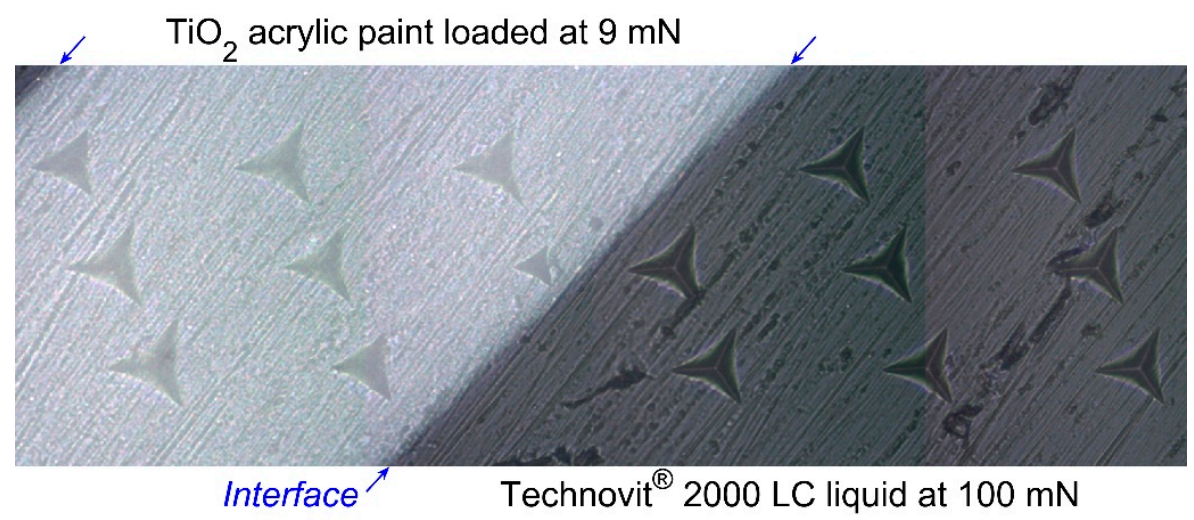

Figure 10. Optical microscope images of Berkovich imprints on the cross-sections of $0.26 \mathrm{~mm}$-thick $\mathrm{TiO}_{2}$ acrylic paint film (white region) and surrounding Technovit ${ }^{\circledR} 2000 \mathrm{LC}$ liquid as the embedding material (dark region), loaded at 9 and $100 \mathrm{mN}$, respectively.

Infiltration of the embedding resin is not the only factor responsible for the overestimation of the measured hardness near the paint-resin interface. Indentation tests performed on an embedded, submillimeter cross-sectional paint sample may also overestimate mechanical properties due to the presence of surrounding resin that is stiffer than the paint itself $[48,49]$. The extent of paint modulus overestimation is greater at test locations closer to the interface with a stiffer embedding material and can be further exacerbated by infiltration of the embedding resin into the paint sample. Such a phenomenon (possible when the sample is porous, and the embedding material is liquid-like) irreversibly changes the composition of the paint material. The simplest approach to avoiding both edge and infiltration effects is to perform measurements away from the sample-resin interface. This approach, however, is impractical for minute cross-sectional samples especially because the impact from the edge and infiltration effects is difficult to assess for samples of unknown mechanical properties. The dilemma is well illustrated in [14] where the authors practically exhausted the available surface area of a small Van Gogh paint sample to maximize the number of indents and obtain as much information as possible about the paint properties.

While mitigating both the edge and infiltration effects in an embedded paint sample may be difficult or impossible, use of an appropriate contact mechanics model may account for the edge effect alone (i.e., in the absence of infiltration). Using the concept of structural compliance proposed by Jakes et al. [49], a method to correct for the edge effect in dynamic sharp indentation was developed and applied to a viscoelastic paint film embedded in a noninfiltrating, light-curing fixing paste [17]. The method established the correct modulus value near the edge of a $\mathrm{TiO}_{2}$ acrylic paint sample embedded in a 10 times stiffer medium. The only assumption used in this method is that the modulus of the paint does not change with depth from the surface. This assumption is reasonable, since a gradient in paint properties is expected in the direction of the film thickness, but not parallel to the film surface.

The decision to employ a noninfiltrating embedding medium for the preparation of paint cross-sections typically means selection of a resin with an even greater stiffness mismatch between the paint and the resin in cured condition. For example, the storage modulus of light-cured Technovit ${ }^{\circledR} 2000$ LC liquid is equal to $\sim 4 \mathrm{GPa}$ [16], whereas the storage modulus of light-cured Technovit ${ }^{\circledR} 2000$ LC fixing paste is 15 GPa [17]. A larger difference between the moduli of paint and resin results in a more pronounced edge effect, but this may be mitigated by use of the previously discussed structural compliance method. 


\subsubsection{Measuring Gradients in Material Properties}

While the main advantage of preparing samples in a cross-sectional form is to enable testing of small volumes of original historic materials, its specific sample orientation provides the added benefit of allowing examination of gradients of mechanical properties across the paint thickness. Due to interactions with the environment and the surrounding materials, the physical properties of paints films can vary from surface to bulk. Drying and aging of the paint itself can also result in the formation of a 'skin surface' or uneven distribution of pigment particles across the paint thickness [50,51]. This poses a challenge when interpreting the results of indentation on free film samples. As discussed earlier, sharp indentation of a free paint film beyond a certain depth becomes inevitably affected by the substrate. The method to define this critical depth relies on identifying the range of indentation depths in which the measured modulus remains constant. This, however, requires that the properties of the paint remain unchanged within the depth range of the test.

In contrast, cross-sectional samples present a gradient of properties on the sample surface due to mounting in resin of a sliver of paint film in a perpendicular orientation. Thus, the properties of a tested material vary with the distance from the paint-resin interface, but not with indentation depth. For such systems, the intrinsic modulus of the paint material may be obtained using the method developed in [17], which analyzes the structural compliance responsible for the distortion of the stiffness versus indentation depth curve.

\section{Summary}

The complexity of analyzing the mechanical properties of historic and artists' paints stems from two separate issues. First, the intrinsic properties of the pigment-binder composite material may vary due to its heterogeneity, time-dependent behavior, and sensitivity to temperature and relative humidity. Testing such materials requires a statistical approach to address a heterogeneous composition and a consistent method to establish the contact depth and, consequently, the probe-material contact area. Second, the analysis of mechanical properties may be impacted by the specific geometry of paint samples and, in the case of historic paints, their small volumes. High surface roughness combined with the substrate effect requires a careful selection of indentation depths. Examination of cross-sectional samples must also consider edge effects. Several research teams addressed these issues by proposing specific measuring protocols and use of indentation probes with various geometries. In addition, it has been shown that sample preparation is an inherently important component of the performed studies.

Common among all the experimental paint studies discussed in this article are the relatively large indentation depths, up to several microns, necessary for testing heterogeneous samples with rough surfaces. Despite these large indentation depths, analysis with high spatial resolution is still needed to account for subtle effects from paint-resin interface and gradients of mechanical properties. Thus, it is particularly important to perform a CSM using a displacement amplitude that is a small fraction of the indentation depth. All nanoindentation studies of artists' paints described in this review paper employed microindentation depths with nanoscale resolution.

Mechanical characterization of artists' paints requires the application of customized measurement protocols and often unconventional methods of data analysis. These are inspired by classic studies of metals, as well as less conventional examinations of polymers and biological tissues. It is hoped that drawing upon research into the latter categories will help bridge the gap between nanoindentation and AFM (commonly used on softer materials), and ultimately allow the quantification of the influence of adhesion on the measured mechanical properties of extremely soft and viscoelastic paints.

Research conducted over the past two decades shows that, while nanoindentation is a well-established method in engineering and materials science, its potential for the micromechanical characterization of historic and artists' paints has yet to be fully realized in the field of cultural heritage. In principle, nanoindentation is capable of measuring 
properties such as yield stress, strength, fracture toughness and residual stress, all of which are needed to model paint behavior in unstable climatic conditions or under external loads. Nanoindentation can be especially effective in tracing subtle changes of material properties. The results obtained are less affected by uncertainty in determining contact dimension and substrate/edge effect when interpreted in a comparative sense, and therefore can be an excellent source of information on the effects of treatments and preventive conservation measures. Consideration of a wide variety of existing indentation parameters, including available probe geometries, context-specific testing protocols, and the use of advanced analytical and numerical models of contact mechanics, provides a rich environment for further exploration by heritage conservation scientists.

Author Contributions: Conceptualization, M.Ł.; writing-original draft preparation, M.Ł., N.F.; writing-review and editing, M.Ł., A.B., N.F.; visualization, N.F., A.B.; supervision, M.Ł., N.F.; All authors have read and agreed to the published version of the manuscript.

Funding: This research received no external funding.

Institutional Review Board Statement: Not applicable.

Informed Consent Statement: Not applicable.

Acknowledgments: The authors would like to acknowledge the support of The Getty Conservation Institute's Managing Collections Environments Initiative. We would also like to thank Vincent Beltran for proofreading the manuscript.

Conflicts of Interest: The authors declare that they have no conflict of interest.

\section{References}

1. Rogala, D.V.; DePriest, P.T.; Charola, A.E.; Koestler, R.J. The Mechanics of Art Materials and Its Future in Heritage Science; Smithsonian Scholarly Press: Washington, DC, USA, 2019.

2. $\quad$ Łukomski, M.; Druzik, J.; Beltran, V.; Freeman, A.; Boersma, F.; Taylor, J. The role of Micromechanics in the Epidemiology of Climate-induced Damage. Mech. Art Mater. Its Future Herit. Sci. 2016, 24-25, 155258.

3. Fischer-Cripps, A. Introduction to Contact Mechanics, 2nd ed.; Springer: Boston, MA, USA, 2007.

4. Fischer-Cripps, A.C. Nanoindentation, 3rd ed.; Springer: New York, NY, USA, 2011.

5. Chiantore, O.; Scalarone, D. The Macro- and Microassessment of Physical and Aging Properties in Modern Paintsof Physical and Aging Properties in Modern Paints. In Proceedings of the Modern Paints Uncovered: Proceedings from the Modern Paints Uncovered Symposium, London, UK, 16-19 May 2006; Getty Publications: Los Angeles, CA, USA, 2007.

6. Wright, M.S.; Hudson, M.; Kokkori, M.; Muir, K.; Casadio, F.; Faber, K.T.; Schull, K.R. Quantifying the mechanical properties of artists' paints with nanoindentation. In Proceedings of the 2014 Annual Meeting of the Adhesion Society, San Diego, CA, USA, 23-26 February 2014.

7. Oliver, W.C.; Pharr, G.M. An improved technique for determining hardness and elastic modulus using load and displacement sensing indentation experiments. J. Mater. Res. 1992, 7, 1564-1583. [CrossRef]

8. Sturdy, L.F.; Wright, M.S.; Yee, A.; Casadio, F.; Faber, K.T.; Shull, K.R. Effects of zinc oxide filler on the curing and mechanical response of alkyd coatings. Polymer 2020, 191, 122222. [CrossRef]

9. Depolo, G.; Walton, M.; Keune, K.; Shull, K.R. After the paint has dried: A review of testing techniques for studying the mechanical properties of artists' paint. Herit. Sci. 2021, 9, 68. [CrossRef]

10. Kaszowska, Z.; Kot, M.; Białek-Kostecka, D.; Forczek-Sajdak, A. Application of micro-indentation tests to assess the consolidation procedure of historic wall paintings. J. Cult. Herit. 2019, 36, 286-296. [CrossRef]

11. Andersen, C.K.; Freeman, A.; Mortensen, M.N.; Beltran, V.; Łukomski, M.; Phenix, A. Mechanical and Moisture Sorption Properties of Commercial Artists' Oil Paint by Dynamic Mechanical Thermal Analysis (DMA), Nanoindentation, and Dynamic Vapour Sorption (DVS). In Conservation of Modern Oil Paintings; Springer: Cham, Switzerland, 2019; pp. $403-418$.

12. Freeman, A.A.; Lee, J.; Andersen, C.K.; Fujisawa, N.; Łukomski, M.; Ormsby, B. A pilot study of solvent-based cleaning of yellow ochre oil paint: Effect on mechanical properties. Herit. Sci. 2021, 9, 28. [CrossRef]

13. Tiennot, M.; Paardekam, E.; Iannuzzi, D.; Hermens, E. Mapping the mechanical properties of paintings via nanoindentation: A new approach for cultural heritage studies. Sci. Rep. 2020, 10, 7924. [CrossRef]

14. Salvant, J.; Barthel, E.; Menu, M. Nanoindentation and the micromechanics of Van Gogh oil paints. Appl. Phys. A 2011, 104, 509-515. [CrossRef]

15. Freeman, A.A.; Fujisawa, N.; Bridarolli, A.; Bertolin, C.; Łukomski, M. Microscale Physical and Mechanical Analyses of Distemper Paint: A Case Study of Eidsborg Stave Church, Norway. Stud. Conserv. 2021, 1-14. [CrossRef] 
16. Freeman, A.; Łukomski, M.; Beltran, V. Mechanical characterization of a cross-sectional TiO2 acrylic-based paint by nanoindentation. J. Am. Inst. Conserv. 2020, 59, 27-39. [CrossRef]

17. Fujisawa, N.; Łukomski, M. Nanoindentation near the edge of a viscoelastic solid with a rough surface. Mater. Des. 2019, 184, 108174. [CrossRef]

18. Oliver, W.C.; Pharr, G.M. Measurement of hardness and elastic modulus by instrumented indentation: Advances in understanding and refinements to methodology. J. Mater. Res. 2004, 19, 3-20. [CrossRef]

19. Fujisawa, N.; Bronken, I.A.T.; Freeman, A.A.; Łukomski, M. Nanoindentation of softening modern oil paints. J. Mech. Phys. Solids 2021. submitted.

20. Tiennot, M.; Iannuzzi, D.; Hermens, E. Evolution of the viscoelastic properties of painting stratigraphies: A moisture weathering and nanoindentation approach. Herit. Sci. 2021, 9, 77. [CrossRef]

21. Giorgiutti-Dauphiné, F.; Pauchard, L. Painting cracks: A way to investigate the pictorial matter. J. Appl. Phys. 2016, 120, 065107. [CrossRef]

22. Sneddon, I.N. The relation between load and penetration in the axisymmetric boussinesq problem for a punch of arbitrary profile. Int. J. Eng. Sci. 1965, 3, 47-57. [CrossRef]

23. King, R. Elastic analysis of some punch problems for a layered medium. Int. J. Solids Struct. 1987, 23, 1657-1664. [CrossRef]

24. Nohava, J.; Randall, N.; Conté, N. Novel ultra nanoindentation method with extremely low thermal drift: Principle and experimental results. J. Mater. Res. 2009, 24, 873-882. [CrossRef]

25. Odegard, G.; Gates, T.; Herring, H. Characterization of viscoelastic properties of polymeric materials through nanoindentation. Exp. Mech. 2005, 45, 130-136. [CrossRef]

26. White, C.C.; VanLandingham, M.R.; Drzal, P.L.; Chang, N.-K.; Chang, S.-H. Viscoelastic characterization of polymers using instrumented indentation. II. Dynamic testing. J. Polym. Sci. Part B Polym. Phys. 2005, 43, 1812-1824. [CrossRef]

27. Herbert, E.G.; Oliver, W.C.; Pharr, G.M. Nanoindentation and the dynamic characterization of viscoelastic solids. J. Phys. D Appl. Phys. 2008, 41, 074021. [CrossRef]

28. Hay, J.; Agee, P.; Herbert, E. Continuous stiffness measurement during instrumented indentation testing. Exp. Tech. 2010, 34, 86-94. [CrossRef]

29. Lucas, B.N.; Oliver, W.C. Indentation power-law creep of high-purity indium. Met. Mater. Trans. A 1999, 30, 601-610. [CrossRef]

30. Tabor, D. The Hardness of Metals; Clarendon: Oxford, UK, 1951; Chapter 2.

31. Bolshakov, A.; Pharr, G.M. Influences of pileup on the measurement of mechanical properties by load and depth sensing indentation techniques. J. Mater. Res. 1998, 13, 1049-1058. [CrossRef]

32. Field, J.; Swain, M. A simple predictive model for spherical indentation. J. Mater. Res. 1993, 8, 297-306. [CrossRef]

33. Kalidindi, S.; Pathak, S. Determination of the effective zero-point and the extraction of spherical nanoindentation stress-strain curves. Acta Mater. 2008, 56, 3523-3532. [CrossRef]

34. Keck, S. Mechanical alteration of the paint film. Stud. Conserv. 1969, 14, 9-30.

35. Mecklenburg, M.F.; Tumosa, C.S.; Erhardt, D. The changing mechanical properties of aging oil paints. In Proceedings of the Materials Issues in Art and Archaeology VII: The Materials Research Society Symposium, Boston, MA, USA, 29 November-2 December 2004.

36. Mecklenburg, M.F.; Tumosa, C.S.; Vicenzi, E.P. The influence of pigments and ion migration on the durability of drying oil and alkyd paints. In New Insights into the Cleaning of Paintings, Proceedings of the Cleaning 2010 International Conference, Universidad Politecnica de Valencia and Museum Conservation Institute, Valencia, Spain, 26-28 May 2010; Smithsonian Institution: Washington, DC, USA, 2013.

37. Fuster-López, L.; Izzo, F.C.; Damato, V.; Yusá-Marco, D.J.; Zendri, E. An insight into the mechanical properties of selected commercial oil and alkyd paint films containing cobalt blue. J. Cult. Heritage 2019, 35, 225-234. [CrossRef]

38. Sandu, I.C.A.; Schäfer, S.; Magrini, D.; Bracci, S.; Roque, C.A. Cross-section and staining-based techniques for investigating organic materials in painted and polychrome works of art: A review. Microsc. Microanal. 2012, 18, 860-875. [CrossRef]

39. Karpowicz, A. A study on development of cracks on paintings. J. Am. Inst. Conserv. 1990, 29, 169-180. [CrossRef]

40. Michalski, S. Crack Mechanisms in Gildin; Gilded Wood Conservation and History Journal: Madison, CT, USA, $1991 ;$ pp. 171-181.

41. Mecklenburg, M.F. Some mechanical and physical properties of gilding gesso. In Gilded Wood Conservation and Histor; Bigelow, D., Cornu, E., Landrey, G.J., van Horne, C., Eds.; Sound View Press: Madison, CT, USA, 1991; pp. 163-170.

42. Krzemień, L.; Łukomski, M.; Bratasz, Ł.; Kozłowski, R.; Mecklenburg, M.F. Mechanism of craquelure pattern formation on panel paintings. Stud. Conserv. 2016, 61, 324-330. [CrossRef]

43. Hay, J. Introduction to Instrumented Indentation Testing. Exp. Tech. 2009, 33, 66-72. [CrossRef]

44. Bronken, I.A.T.; Boon, J.J. Hard Dry Paint, Softening Tacky Paint, and Exuding Drips on Composition (1952) by Jean-Paul Riopelle. In Issues in Contemporary Oil Paint; Springer: Singapore, 2014; pp. 247-262.

45. Van den Berg, K.J.; Burnstock, A.; de Keijzer, M.; Krueger, J.; Learner, T.; de Tagle, A.; Heydenreich, G. Issues in Contemporary Oil Paint; Springer: New York, NY, USA, 2014.

46. Cao, Y.; Ma, D.; Raabe, D. The use of flat punch indentation to determine the viscoelastic properties in the time and frequency domains of a soft layer bonded to a rigid substrate. Acta Biomater. 2009, 5, 240-248. [CrossRef] [PubMed]

47. Khandekar, N. Preparation of cross-sections from easel paintings. Stud. Conserv. 2003, 48, 52-64. [CrossRef] 
48. Jakes, J.; Frihart, C.; Beecher, J.; Moon, R.; Stone, D. Experimental method to account for structural compliance in nanoindentation measurements. J. Mater. Res. 2008, 23, 1113-1127. [CrossRef]

49. Jakes, J.; Stone, D.S. The edge effect in nanoindentation. Philos. Mag. 2011, 91, 1387-1399. [CrossRef]

50. Holl, Y.; Keddie, J.L.; McDonald, P.J.; Winnik, W.A. Drying Modes of Polymer Colloids. ACS Symp. Ser. 2001, 790, 2-26.

51. Learner, T.J.S.; Smithen, P.; Krueger, J.W.; Michael, R. Modern Paints Uncovered. In Proceedings of the Modern Paints Uncovered Symposium, London, UK, 16-19 May 2007; Getty Publications: Los Angeles, CA, USA, 2007. 